(J. Bact., 95, 469; 1968) have tested whether this sequential enzyme synthesis of outgrowth is in fact a reflexion of an ordered transcription or, alternatively, whether it is a manifestation of continuous induction or derepression in which an ordered synthesis of enzymes is made by cyclic variations in repressor and corepressor levels. In Bacillus cereus $\mathrm{T}$, the times of $\alpha$-glucosidase, L-alanine dehydrogenase and histidase syntheses were ordered and commenced at specific times after the initiation of germination, approximately 10,15 and $35 \mathrm{~min}$ respectively. Significantly the timing of these syntheses was not influenced by induction or repression conditions; if the genome was available for transcription continually throughout outgrowth, the enzyme periodicity should have been disturbed.

The conclusion that ordered enzyme synthesis resulted from an ordered transcription sequence was confirmed by studies of enzyme synthesis in the presence of actinomycin $D$. a-Glucosidase synthesis was stopped within 3-4 min of the addition of the actinomycin $D$. This lifetime of enzyme messenger is identical to the lifetime of pulse-labelled RNA at the same period of ontogeny. The kineties of the histidase induction were very similar to those of $a$-glucosidase. The order of enzymes synthesized during outgrowth was identical to that observed in the first few synchronous divisions of vegetative growth. By contrast, the genome appears to be available continuously for induction in synchronized vegetative cultures of $B$. subtilis and Escherichia coli. Steinberg and Halvorson suggest the timing of enzyme synthesis in vegetative cells of $B$. subtilis and germinated spores of $B$. cereus is under different control systems: possibly regulation in outgrowth is rigidly controlled but, after outgrowth is finished, the regulation mechanism becomes relaxed.

In a second publication (J. Bact., 95, 479; 1968), the same authors report the results of experiments designed to test whether ordered transcription is controlled by DNA replication. Spore outgrowth provides an ideal system for this test because the initial stage of outgrowth proceeds synchronously in the absence of net DNA synthesis and is dependent on de novo $m$ RNA transcription. Evidence was obtained which proves that, during outgrowth, transcription is not controlled by DNA replication. In particular, addition of thymine to a thymine auxotroph did not affect the periodicity of a-glucosidase and histidase. To investigate how ordering might be achieved in the absence of DNA replication, experiments were done with mitomycin $C$ and 5-fluoro-2'-deoxyuridine (FUdR). At first glance the results from mitomycin $C$ experiments appear to conflict with findings with the thymine auxotroph. However, the use of FUdR (an experimental system which mimics the conditions used with the thymine auxotroph) substantiated the induction of enzymes to control levels even though DNA synthesis was only 20 per cent of the control level. The authors propose, to explain the anomalous result with mitomycin $C$, that the drug induces some physical modification of the DNA (presumably cross-linking) that impairs transcription.

\section{More Coiled Coils and Catenanes}

\section{from our Molecular Biology Correspondent}

SINCE I discussed, two weeks ago, recent work on the supercoiling of circular DNA, three further studies from Vinograd's laboratory have appeared. The first (Proc. US Nat. Acad. Sci., 59, 838; 1968) concerns the mitochondrial DNA of sea urchin eggs, which in these cells makes up the greater part of the total DNA. The DNA was isolated by the ethidium bromide density gradient method, in which linear DNA takes up more dye than circular DNA, and consequently bands in a different position on caseium chloride density gradient sedimentation. Three bands were in fact observed in this system, one containing linear molecules, both mitochondrial and nuclear, the other two only circular species. In the one (the lower band) 5 per cent of the molecules were linked circles, or catenanes, whereas the smallest (middle) band contained 63 per cent of such dimers, as well as 3 per cent of trimers and $1 \cdot 2$ per cent of tetramers. The intermediate position of this band was taken to mean that one of the two circles of the dimers in each case contained a single-chain break and was therefore able to take up more dye than its partner, thus yielding a dimer of intermediate density. Overall, the total proportion of dimers in the mitochondrial DNA, measured by electron microscopy in a population of 1,300 molecules, was 11 per cent, with 1 per cent of trimers and 0.3 per cent of tetramers. The total contour length of a single circle was very uniform, being a little less than $5 \mu$. These remarkable catenated molecules have previously been observed in HeLa cells and in leucocytes.

Bauer and Vinograd (J. Mol. Biol., 33, 141; 1968) have described in greater detail the conformational behaviour of circular viral $(S V 40)$ DNA on addition of intercalating dye. Their earlier results have now been placed on a more quantitative footing; thus, under standard conditions of high salt concentration, the DNA has some 14 turns of left-handed superhelix. On addition of the dye, ethidium bromide, the partial untwisting of double-helical structure, which is occasioned by intercalation, leads progressively to unwinding of the super helix, and rewinding in the opposite (right-handed) sense, up to apparently a maximum of about 45 turns. By contrast, a circular DNA containing a single-strand break does not supercoil, and undergoes no topological change with uptake of dye. The unbroken DNA initially takes up dye with higher affinity, so as to release the strain of supercoiling, but as the dye concentration increases, so the affinity diminishes in comparison with that of once-broken DNA.

The unwinding of the superhelix on denaturation of the DNA is considered in the succeeding paper (Vinograd et al., ibid., 173). As also observed by the Dutch group working on a similar molecule, the early stages of alkaline denaturation lead to unwinding. This initial transition involves the titration of only some 3 per cent of the bases, and is accompanied by a considerable change in buoyant density. It is assisted by the release of the energy of supercoiling, and therefore precedes the denaturation of unsupercoiled DNA. Further denaturation, however, generates more slack, which must initially be taken up by formation of new turns of right-handed supercoil, so that this processthe main transition - is inhibited relative to the linear or once-broken forms. The thermal melting temperature of the supercoiled DNA is likewise elevated-in the one solvent it is $25^{\circ} \mathrm{C}$ higher than that of circular DNA containing one broken bond. 